\title{
Anesthetic Experience of Spinal Anesthesia after Sedation in Un-cooperated Elderly Patients
}

\author{
Bon-Sung Koo, Myung-Jin Jung, Joon-Ho Lee, Sung-Hwan Cho, Sang-Hyun Kim, Won-Seok Chae \\ Department of Anesthesiology and Pain Medicine, Soonchunhyang University Bucheon Hospital, Soonchunhyang University College of Medicine, Bucheon, \\ Korea
}

Regional anesthesia is mostly used in operations on the lower abdomen or lower extremities in elderly patients. It shows nearly no difference in long-term outcomes compared to general anesthesia, but it is used more often because of the several advantages. However, during the regional anesthetic procedures, the patient must cooperate and has to be lateral decubitus position without physical movement. Therefore, in the case of the patients who are not cooperated, it may be not easy to perform regional anesthesia. In this study, we present 3 case reports that regional anesthesia after sedation is performed in un-cooperated patients. Regional anesthesia after sedation may be a good method to improve outcomes in un-cooperated elderly patients.

Keywords: Bispectral index monitors; Sedation; Spinal anesthesia

\section{INTRODUCTION}

According to the data reported by Statistics Korea, current elderly population over the age of 65 is 6.62 million [1]. This means 100 productive Koreans must support 17.9 elderly people over the age of 65 . With the development of medicine and social welfare in Korea, it has become one of the countries with the fastest growth in its elderly population. Due to such increase, the number of its elderly patients is also believed to be increasing. And currently, even elderly patients over the age of 85 can be easily seen undergoing operations.

Regional anesthesia which is mostly used in operations on the lower abdomen or lower extremities in elderly patients shows nearly no difference in long-term outcome compared to general anesthesia [2]. However, regional anesthesia is used more often for elderly patients because it has the advantages of reducing blood loss, decreasing risk of embolism, and maintaining the lung function $[3,4]$.

During the regional anesthetic procedures, the patient must cooperate and have to be lateral decubitus position without physical movement. But there are some elderly patients who cannot be co- operated due to various problems, such as dementia, excessive anxiety, etc. In these patients, some have a high risk to do general anesthesia. Therefore, we consider to perform regional anesthesia after sedation.

We report cases of successful regional anesthesia after sedation in elderly patients who are not cooperated.

\section{CASE REPORTS}

\section{Case 1}

An 87-year-old man with $167 \mathrm{~cm}$ height and $51 \mathrm{~kg}$ weight was admitted to the department of orthopedics for left femur neck fracture. He was scheduled to undergo bipolar hemiarthroplasty. The patient had a history of hypertension, ischemic heart disease, end-stage renal disease, and dementia, and he was undergoing dialysis three times a week.

Preoperative hemoglobin and hematocrit were $6.8 \mathrm{~g} / \mathrm{dL}$ and $20.7 \%$, respectively. After blood transfusion, they were $8.0 \mathrm{~g} / \mathrm{dL}$ and $25.2 \%$, respectively. Preoperative platelet count was normal. Blood test for blood urea nitrogen and creatinine showed $10.3 \mathrm{mg} /$ $\mathrm{dL}$ and $3.0 \mathrm{mg} / \mathrm{dL}$, respectively after dialysis. His chest X-ray re- 
Koo BS, et al. • Spinal Anesthesia after Sedation

Table 1. Perioperative bispectral index and vital signs

\begin{tabular}{|c|c|c|c|c|c|c|c|}
\hline \multirow{2}{*}{ Case } & \multirow{2}{*}{ Variable } & \multicolumn{6}{|c|}{ Time } \\
\hline & & $\mathrm{T}_{0}$ & $\mathrm{~T}_{\mathrm{S}}$ & $\mathrm{T}_{30}$ & $\mathrm{~T}_{60}$ & $\mathrm{~T}_{90}$ & $T_{p}$ \\
\hline \multirow[t]{4}{*}{ Case 1} & BIS & 95 & 78 & 78 & 75 & 73 & \\
\hline & Blood pressure $(\mathrm{mmHg})$ & $164 / 77$ & $181 / 63$ & $126 / 41$ & $107 / 46$ & $99 / 46$ & $127 / 49$ \\
\hline & Heart rate (bpm) & 95 & 97 & 87 & 74 & 79 & 76 \\
\hline & $\mathrm{O}_{2}$ saturation $(\%)$ & 95 & 96 & 100 & 100 & 99 & 100 \\
\hline \multirow[t]{4}{*}{ Case 2} & BIS & 94 & 74 & 77 & 74 & 79 & \\
\hline & Blood pressure $(\mathrm{mmHg})$ & $160 / 82$ & $153 / 84$ & $129 / 54$ & $122 / 52$ & $115 / 49$ & $131 / 66$ \\
\hline & Heart rate (bpm) & 55 & 54 & 51 & 49 & 46 & 52 \\
\hline & $\mathrm{O}_{2}$ saturation (\%) & 97 & 100 & 100 & 100 & 100 & 99 \\
\hline \multirow[t]{4}{*}{ Case 3} & BIS & 90 & 71 & 73 & 75 & 73 & \\
\hline & Blood pressure $(\mathrm{mmHg})$ & $132 / 60$ & $106 / 49$ & $80 / 33$ & $95 / 43$ & $82 / 38$ & $85 / 52$ \\
\hline & Heart rate (bpm) & 73 & 74 & 68 & 70 & 62 & 75 \\
\hline & $\mathrm{O}_{2}$ saturation $(\%)$ & 95 & 97 & 96 & 99 & 99 & 95 \\
\hline
\end{tabular}

BIS, bispectral index; $T_{0}$ operation room admission; $T_{s}$, after spinal anesthesia; $T_{30}, 30$ minutes after $T_{S} ; T_{60}, 60$ minutes after $T_{s} ; T_{90}, 90$ minutes after $T_{s} ; T_{p}$ at post anesthesia care unit.

vealed pleural effusion in right lung, and he showed slight tachypnea. He was unable to perform the pulmonary function test due to uncooperation. Electrocardiogram (ECG), echocardiogram, and other laboratory findings including blood coagulation test were within the normal range.

Premedication was not given. Intraoperative monitors included non-invasive automated blood pressure (BP), ECG, and pulse oximetry $\left(\mathrm{SpO}_{2}\right)$. Pre-anesthetic baseline vital signs were BP 164/77 $(\mathrm{mmHg})$, heart rate (HR) 95 beats per minute (bpm), and $\mathrm{SpO}_{2}$ 95\%. He wouldn't say anything, responded violently to even mild pain, and did not cooperate at all. And so, we decided that sedation was first performed using propofol.

Before the patient was sedated, an anesthesia depth monitor (bispectral index [BIS]-quatro; Aspect Medical Systems Inc., Natick, MA, USA) was attached to forehead of the patient. Continuous intravenous propofol infusion was titrated at $25-100 \mu \mathrm{g} /$ $\mathrm{kg} / \mathrm{min}$ to keep the BIS at 70-80. Since then, $5 \mathrm{~L} / \mathrm{min}$ of oxygen was supplied with a mask. After confirmation of sedation, arterial cannulation was performed right radial artery to monitor invasive arterial BP. After vital signs and sedation status were stable, spinal anesthesia were performed in the lateral decubitus position at the L3-L4 intervertebral space using a 24G Quincke needle via the paramedian approach. Ten mg of $0.5 \%$ heavy bupivacaine and 10 $\mu \mathrm{g}$ of fentanyl were injected into the subarachnoid space. And the patient was maintained in the supine position. For 10 minutes, BP and HR were maintained without significant change.

$\mathrm{BP}, \mathrm{HR}, \mathrm{SpO}_{2}$, BIS were recorded at the following time points: af- ter spinal anesthesia, every 30 minutes after spinal anesthesia, and at post anesthesia care unit (Table 1). There was no significant change in the ECG, BP, and oxygen saturation during the operation except that esmolol $10 \mathrm{mg}$ was injected for the tachycardia. Transfusion was started due to bleeding of about $1,500 \mathrm{~mL}$ pooled blood from the hematoma. At the end of operation, phenylephrine was injected twice for low $\mathrm{BP}$ due to the change in the body position from lateral decubitus to supine. The total operation time was 1 hour 35 minutes, and total anesthesia time was 2 hours 45 minutes. The pain score via numeric rating scale (NRS) was 0 after the operation. And the patient was transferred to the intensive care unit.

The patient complained of no significant problem exception of pain. But, on the 12th postoperative day, he passed away under the diagnosis of aspiration pneumonia due to sudden dyspnea.

\section{Case 2}

An 80-year-old woman with a height of $155 \mathrm{~cm}$ and weigh of 34 $\mathrm{kg}$ was admitted to the department of orthopedics for left femur neck fracture occurring from slip down. She was scheduled to undergo bipolar hemiarthroplasty. About 18 years ago, she underwent an operation for gastric cancer. She had a history of cerebral infarction but had no sequelae, and also had Parkinson's disease and dementia.

Preoperative laboratory, chest radiographic, and ECG findings were within the normal limits. Before entering the operating room, the patient and her family agreed to be performed regional anesthesia. Premedication was not given. After arrival of the pa- 
tient in the operating room, three-lead electrocardiography, noninvasive automated $\mathrm{BP}$, and pulse oximetry $\left(\mathrm{SpO}_{2}\right)$ were monitored. Initial BP was 160/82 mmHg. $\mathrm{HR}$ was $55 \mathrm{bpm}$, and $\mathrm{SpO}_{2}$ was $97 \%$. But it was confirmed that communication with the patient was not possible. Therefore, pre-anesthetic sedation was started by continuous intravenous propofol infusion.

Before the sedation, the BIS was attached to forehead of the patient. Five L/min of oxygen was given to patient via mask. Continuous intravenous propofol infusion was titrated at $25-100 \mu \mathrm{g} / \mathrm{kg} /$ min to keep the BIS at 70-80. And then, invasive arterial BP monitoring and left external jugular vein cannulation were performed. The patient was placed in the left lateral decubitus position, and sterilization and local anesthetics infiltration for injection site of skin was done. A 24G Quincke needle was inserted at the L3-L4 intervertebral space. Eight mg of $0.5 \%$ heavy bupivacaine and $10 \mu$ $\mathrm{g}$ of fentanyl were injected into the subarachnoid space. The patient was then immediately turned to a supine position after the drug administration. And the operation was started after vital signs were stable.

$\mathrm{BP}, \mathrm{HR}, \mathrm{SpO}_{2}$, and BIS were recorded at the following time points: after spinal anesthesia, every 30 minutes after spinal anesthesia, at post anesthesia care unit (Table 1). About 20 minutes after spinal anesthesia, BP and HR were 92/45 mmHg and $66 \mathrm{bpm}$, respectively. Therefore ephedrine $4 \mathrm{mg}$ was injected once. After then, the patient's vital signs were stable during the surgery.

The total operation time was 60 minutes, and the total anesthesia time was 1 hour 50 minutes. The NRS score was 0 at end of operation, and the patient was transferred to the general ward with no complication. The patient was discharged without any hemodynamic instability or cardiovascular complications on postoperative day 18 .

\section{Case 3}

An 82-year-old woman, $173 \mathrm{~cm}$ height and $63 \mathrm{~kg}$ weight, came to the orthopedics department. The patient was diagnosed with right femur neck fracture. She was scheduled to undergo closed reduction and internal fixation with proximal femur nail anti-rotation.

The patient had a history of a hypertension, diabetes mellitus, dementia, and myocardial infarction. Due to dementia, communication to patient could not be possible. Preoperative hemoglobin was $9.5 \mathrm{~g} / \mathrm{dL}$, hematocrit was $28.0 \%$, blood sugar was $126 \mathrm{mg} / \mathrm{dL}$, and other laboratory findings were normal. The chest X-ray showed cardiomegaly, and the ECG showed atrial fibrillation. Be- cause of these previous history and general condition, the patient's guardian strongly desired regional anesthesia. And we fully explained relation of regional anesthesia and sedation and the possibility of general anesthesia if needed.

Premedication was not given. After arrival of the patient in the operating room, standard monitoring (non-invasive automated $\mathrm{BP}, \mathrm{ECG}$, and $\mathrm{SpO}_{2}$ ) was begun. Initial $\mathrm{BP}$ was 132/60 mmHg, $\mathrm{HR}$ was $73 \mathrm{bpm}$, and $\mathrm{SpO}_{2}$ was 95\%. The BIS was attached to forehead of the patient, and then sedation was started. At the same time, 5 $\mathrm{L} / \mathrm{min}$ of oxygen was supplied to patient with a mask. Continuous intravenous propofol infusion was titrated at $25-100 \mu \mathrm{g} / \mathrm{kg} / \mathrm{min}$ to keep the BIS at 70-80. Arterial cannulation was performed in left radial artery to monitor invasive arterial BP. The patient was placed in the right lateral decubitus position, and sterilization and local anesthetics infiltration for injection site of skin was done. A 24G Quincke needle was inserted at the L3-L4 intervertebral space via the paramedian approach. Ten $\mathrm{mg}$ of $0.5 \%$ heavy bupivacaine and $10 \mu \mathrm{g}$ of fentanyl were injected into the subarachnoid space. After she was turned to a supine position, BP and HR were maintained without significant change for 10 minutes.

$\mathrm{BP}, \mathrm{HR}, \mathrm{SpO}_{2}$, and BIS were recorded at the following time points: after spinal anesthesia, every 30 minutes after spinal anesthesia, and at post anesthesia care unit (Table 1). At 30 minutes after spinal anesthesia, hypotension (BP, 80/33 mmHg) developed, and $6 \mathrm{mg}$ of ephedrine was administrated once. Since then, BP was maintained at 90-110 $\mathrm{mmHg} / 40-60 \mathrm{mmHg}$. There was no significant change in the ECG and there was no hypoxemia during the operation.

The total operation time was 60 minutes, and anesthesia time was 2 hours. The pain score via NRS was 0 after the operation, and the patient was transferred to the general ward with no additional episodes. On postoperative day 4, the patient showed no significant symptom. But, in laboratory findings, D-dimer level was elevated to $4.46 \mathrm{mg} / \mathrm{L}$ (normal range, 0 to $0.65 \mathrm{mg} / \mathrm{L}$ ). Under the diagnosis of suspicious pulmonary embolism due to deep vein thrombosis, further evaluation was done. However, follow-up laboratory findings were within normal limits without any treatment. The patient was discharged 19 days postoperatively without specific complications.

\section{DISCUSSION}

In elderly patients, regional anesthesia is one of commonly used 
methods in orthopedic and urologic surgery. Generally speaking, it is difficult to conclude that regional anesthesia is superior to general anesthesia. However, a large number of studies have shown the advantages of regional anesthesia in the short term outcome. According to Fields et al. [5], in observation of 6,133 patients who underwent hip fracture surgery, general anesthesia was shown to have a higher risk of thirty-day complications than regional anesthesia. Lee et al. [6] reported six cases (1.4\%) of pulmonary embolism for orthopedic surgery in 422 patients older than 80 years, which all occurred under general anesthesia. Based on these cases, regional anesthesia can be generally preferred for elderly patients.

Sedation during regional anesthesia decreases anxiety and stress from the surgery; therefore, anesthesia stabilizes the cardiovascular system. And it produced a high degree of patient satisfaction in the postoperative phase. Propofol is the most commonly used intravenous anesthetics and sedatives these days, showing a rapid sedative effect and recovery. Furthermore, propofol has a antiemetic effect and provide a general sensation of well-being $[7,8]$. Also, spinal anesthesia itself was found be reduce the dosage of sedative drugs [9].

BIS is an anesthetic depth monitoring device that analyzes the electroencephalogram and directly measures the consciousness of the patient. It was made to measure the hypnotic effect of the anesthetic and ranges from 0 to 100 [10]. According to Glass et al. [11], the BIS value at which $95 \%$ of patients had a loss of recall were 77 when using propofol. As a similar results, our finding suggest that sedation and complete loss of recall are also performed well in elderly patients without abnormal cardiopulmonary response.

Intraoperative hypotension is one of a common physiologic symptom caused by neuraxial block, which is more common in elderly patients than young patients [12]. Intravenous volume loading and vasopressors can be administered to treat or prevent hypotension. However, prophylactic fluid administration is no longer used as conventional methods [13]. Therefore, in order to avoid profound hypotension and high level sympathectomy, we are trying to maintain the adequate sensory blockade and sympathectomy with using a smaller amount of local anesthetics and opioid mixture. According to Kim et al. [14], the range of sensory block levels showed T7-T10 with 8-9 mg of 0.5\% hyperbaric bupivacaine in elderly patients. But our anesthesiology department experienced that several cases of patients complained of pain when using the above dosage for operations lasting more than 90 min- utes. Therefore, when we performed a spinal anesthesia, according to the weight and height, $10-12 \mathrm{mg}$ of $0.5 \%$ hyperbaric bupivacaine and 10-20 $\mu$ g of fentanyl have been injected into the subarachnoid space in elderly male patients. On the other hand, $8-10 \mathrm{mg}$ of $0.5 \%$ hyperbaric bupivacaine and 10-20 $\mu \mathrm{g}$ of fentanyl have been administrated in elderly female patients. The level of sensory blockade showed a T8-T12 level and was kept for at least 120 to 150 minutes. And the temporary hypotension was easily restored through the administration of ephedrine or phenylephrine. Small amount of intrathecal fentanyl as a means of prolonging spinal anesthesia is localized to the injected site, which does not increase the sensory block level. In addition, it potentiates the analgesic effect and reduces the hypnotic requirement of propofol [15].

In case 1 , regional anesthesia was more favorable because there was a high risk of pulmonary complication during general anesthesia due to pleural effusion. In case 2, there was no problem in the general condition, but communication was not possible. In case 3, patient's guardian strongly desired regional anesthesia. All three cases are not a contraindication of regional anesthesia and can be yielded the advantages of regional anesthesia, which include a decreasing blood loss and transfusion requirements, preservation of the pulmonary function, and reduction in venous thromboembolic complications. In addition, regional anesthesia after sedation may be recommended for patients who are extremely anxious or for elderly patients who are alert but refuse to communicate. But we have some difficulties in performing regional anesthesia after sedation. First, the level of sensory block cannot be checked precisely using this method. So we injected a pre-determined dose based on empirical methods to maintain the adequate sensory blockade. Second, sedatives-related respiratory depression may be happened. Therefore, we should consider a variety of situations.

In conclusion, we successfully experienced 3 case reports which regional anesthesia after sedation is performed in un-cooperated patients. In elderly patients, there are some patients who have the high risks to do general anesthesia. In that cases, if patients are not cooperated, regional anesthesia after sedation may be a good method to improve outcomes.

\section{REFERENCES}

1. Statistics Korea. 2015 Senior citizen statistics. Daejeon: Statistics Korea; 2015. 
2. Roy RC. Choosing general versus regional anesthesia for the elderly. Anesthesiol Clin North America 2000;18:91-104, vii.

3. Covert CR, Fox GS. Anaesthesia for hip surgery in the elderly. Can J Anaesth 1989;36(3 Pt 1):311-9.

4. Rosenfeld BA, Beattie C, Christopherson R, Norris EJ, Frank SM, Breslow MJ, et al. The effects of different anesthetic regimens on fibrinolysis and the development of postoperative arterial thrombosis. Perioperative Ischemia Randomized Anesthesia Trial Study Group. Anesthesiology 1993; 79:435-43.

5. Fields AC, Dieterich JD, Buterbaugh K, Moucha CS. Short-term complications in hip fracture surgery using spinal versus general anaesthesia. Injury 2015;46:719-23.

6. Lee EH, Do KJ, Yang HS. Anesthesia for orthopedic surgery in patients older than 80 years. Korean J Anesthesiol 2007;52:537-42.

7. Cechetto DF, Diab T, Gibson CJ, Gelb AW. The effects of propofol in the area postrema of rats. Anesth Analg 2001;92:934-42.

8. Pain L, Gobaille S, Schleef C, Aunis D, Oberling P. In vivo dopamine measurements in the nucleus accumbens after nonanesthetic and anesthetic doses of propofol in rats. Anesth Analg 2002;95:915-9.

9. Tverskoy M, Fleyshman G, Bachrak L, Ben-Shlomo I. Effect of bupiva- caine-induced spinal block on the hypnotic requirement of propofol. Anaesthesia 1996;51:652-3.

10. Rampil IJ. A primer for EEG signal processing in anesthesia. Anesthesiology 1998;89:980-1002.

11. Glass PS, Bloom M, Kearse L, Rosow C, Sebel P, Manberg P. Bispectral analysis measures sedation and memory effects of propofol, midazolam, isoflurane, and alfentanil in healthy volunteers. Anesthesiology 1997;86: 836-47.

12. Carpenter RL, Caplan RA, Brown DL, Stephenson C, Wu R. Incidence and risk factors for side effects of spinal anesthesia. Anesthesiology 1992;76:906-16.

13. Loubert C. Fluid and vasopressor management for Cesarean delivery under spinal anesthesia: continuing professional development. Can J Anaesth 2012;59:604-19.

14. Kim YS, Kim DK, Lee KC, Kang PS, Woo NS, Lee YC. Study of spinal sensory block height with $0.5 \%$ heavy bupivacaine in elderly patients. Korean J Anesthesiol 2003;44:488-93.

15. Lee KY, Kwak KH, Kim SO. The effect of intrathecal fentanyl on hypnotic requirement of propofol during spinal anesthesia. Korean J Anesthesiol 2005;49:496-501. 